\title{
Effects of retractors on endotracheal tube cuff pressure and recurrent laryngeal nerve palsy during anterior cervical discectomy and fusion : A preliminary study
}

Kornkamon Yuwapattanawong, M.D., Lawan Tuchinda, M.D., Wanna Somboonviboon, M.D.,

Surunchana Lerdsirisopon, M.D., Sunisa Sangtongjaraskul, M.D.

Department of Anesthesiology, Faculty of Medicine, Chulalongkorn University

King Chulalongkorn Memorial Hospital The Thai Red Cross Society, Bangkok, Thailand

\section{BACKGROUND}

The surgical retractor had been applied to visualise the anterior cervical spine during anterior cervical discectomy and fusion (ACDF). As a result, the recurrent laryngeal nerve was trapped between retractor and endotracheal tube (ETT) cuff. An increasing of ETT cuff pressure with retractor placement might produce recurrent laryngeal nerve palsy. Symptoms included dysphagia, hoarseness and sore throat. The aim of the present study was to observe the effects of retractors and changes in ETT cuff pressure and to evaluate the influence of ETT cuff pressure adjustment after retractor placement on postoperative dysphagia, hoarseness and sore throat.

\section{METHODS}

A prospective randomised and observational study was undertaken with patients scheduled for elective ACDF. Patients were randomised into four groups : group (A) ETT cuff pressure was adjusted and maintained at $20 \mathrm{~mm}$ $\mathrm{Hg}$ after retractor placement without $\mathrm{N}_{2} \mathrm{O}$ anaesthesia, group (B) ETT cuff pressure was not adjusted without $\mathrm{N}_{2} \mathrm{O}$ anaesthesia, group (C) ETT cuff pressure was adjusted and maintained at $20 \mathrm{~mm} \mathrm{Hg}$ with $\mathrm{N}_{2} \mathrm{O}$ anaesthesia and group (D) ETT cuff pressure was not adjusted with $\mathrm{N}_{2} \mathrm{O}$ anaesthesia. ETT cuff pressures and peak airway pressures were continuous recorded. Postoperative dysphagia (the Bazaz dysphagia scale), hoarseness (GRBAS) and sore throat (NRS) were followed up at $24 \mathrm{hr}$ and 1 mo.

\section{RESULTS}

A 24 ASA physical status I-III patients were evaluated. There was significantly increased in ETT cuff pressure values after retractor application when compared to the baseline values in 4 groups. There was no significant difference in changed ETT cuff pressure and peak airway pressure values between $\mathrm{N}_{2} \mathrm{O}$ anesthesia $(C, D)$ and without $\mathrm{N}_{2} \mathrm{O}$ anesthesia (A,B) groups at the same period of time. Postoperative dysphagia, hoarseness and sore throat were found in all patients. The data showed significant lower of the Bazaz dysphagia scale in groups A,C at $24 \mathrm{~h}$ postoperatively $(\mathrm{p}<0.001)$. The severity score of hoarseness (GRBAS) at $24 \mathrm{~h}$ was statistical significant lower score in group A, C than B, D $(p<0.001)$. However, there was no significant difference in the NRS of sore throat.

\begin{tabular}{|c|c|c|c|c|c|}
\hline \multirow[b]{2}{*}{$\begin{array}{c}\text { Paramete } \\
\text { rs }\end{array}$} & \multirow[b]{2}{*}{ Stages } & \multicolumn{4}{|c|}{ Groups } \\
\hline & & A & B & C & D \\
\hline \multirow{4}{*}{$\begin{array}{c}\text { Cuff } \\
\text { pressure } \\
(\mathrm{mm} \mathrm{Hg})\end{array}$} & $\begin{array}{l}\text { Baseline : } \\
\text { T0 }\end{array}$ & $\begin{array}{l}18 \pm 4.5 \\
(12-23)\end{array}$ & $\begin{array}{c}19.5 \pm 2.2 \\
(16-22)\end{array}$ & $\begin{array}{c}18.7 \pm 2.3 \\
(15-21)\end{array}$ & $\begin{array}{c}17.8 \pm 3.1 \\
(15-22)\end{array}$ \\
\hline & $\begin{array}{l}\text { Before } \\
\text { application } \\
\text { of } \\
\text { retractor : } \\
\text { T1 }\end{array}$ & $\begin{array}{c}21.3 \pm 2.1 \\
(19-25) \\
(p=0.207)\end{array}$ & $\begin{array}{c}21.3 \pm 2.0 \\
(20-25) \\
(p=0.066)\end{array}$ & $\begin{array}{c}20.8 \pm 0.8 \\
(20-22) \\
(\mathrm{p}=0.066)\end{array}$ & $\begin{array}{c}20.5 \pm 2.1 \\
(17-23) \\
(p=0.026)\end{array}$ \\
\hline & $\begin{array}{l}\text { During } \\
\text { application } \\
\text { of } \\
\text { retractor: } \\
\text { T2 }\end{array}$ & $\begin{array}{c}24.8 \pm 1.7 \\
(23-28) \\
(\mathrm{p}=0.043)^{*}\end{array}$ & $\begin{array}{c}24.3 \pm 2.9 \\
(22-30) \\
(p=0.043)^{*}\end{array}$ & $\begin{array}{c}24.2 \pm 1.5 \\
(22-26) \\
(p=0.027)^{*}\end{array}$ & $\begin{array}{c}24.8 \pm 1.7 \\
(23-28) \\
(p=0.027)^{*}\end{array}$ \\
\hline & $\begin{array}{l}\text { After } \\
\text { removal of } \\
\text { retractor : } \\
\text { T3 }\end{array}$ & $\begin{array}{c}16.7 \pm 1.5 \\
(15-18) \\
(p=0.465)\end{array}$ & $\begin{array}{c}17.3 \pm 2.0 \\
(16-21) \\
(p=0.336)\end{array}$ & $\begin{array}{c}15.5 \pm 1.4 \\
(13-17) \\
(p=0.058)\end{array}$ & $\begin{array}{c}17.5 \pm 1.8 \\
(15-20) \\
(p=1.000)\end{array}$ \\
\hline
\end{tabular}

\begin{tabular}{|c|c|c|c|c|c|c|c|}
\hline \multirow[b]{2}{*}{$\begin{array}{l}\text { Parameter } \\
\text { s }\end{array}$} & \multirow[b]{2}{*}{$\begin{array}{c}\text { Follow } \\
\text {-up }\end{array}$} & \multirow[b]{2}{*}{ Severity } & \multicolumn{4}{|c|}{ Groups } & \multirow[b]{2}{*}{$\begin{array}{c}P \\
\text { value }\end{array}$} \\
\hline & & & A & B & $\mathrm{C}$ & D & \\
\hline \multirow{8}{*}{$\begin{array}{l}\text { Bazaz } \\
\text { dysphagia } \\
\text { scale }\end{array}$} & \multirow{4}{*}{$24 \mathrm{~h}$} & none & 0 & 0 & 0 & 0 & \multirow{4}{*}{$<0.001$} \\
\hline & & mild & 6 & 0 & 6 & 0 & \\
\hline & & moderate & 0 & 0 & 0 & 1 & \\
\hline & & severe & 0 & 6 & 0 & 5 & \\
\hline & \multirow{4}{*}{$1 \mathrm{mo}$} & none & 4 & 1 & 5 & 3 & \multirow{4}{*}{0.97} \\
\hline & & mild & 2 & 3 & 1 & 1 & \\
\hline & & moderate & 0 & 1 & 0 & 1 & \\
\hline & & severe & 0 & 1 & 0 & 1 & \\
\hline \multirow{4}{*}{$\begin{array}{c}\text { Score of } \\
\text { hoarseness } \\
\text { (GRBAS) }\end{array}$} & \multirow{4}{*}{$24 \mathrm{~h}$} & normal & 0 & 0 & 0 & 0 & \multirow{4}{*}{$<0.001$} \\
\hline & & slight & 6 & 0 & 6 & 0 & \\
\hline & & medium & 0 & 6 & 0 & 6 & \\
\hline & & high & 0 & 0 & 0 & 0 & \\
\hline $\begin{array}{l}\text { Numeric } \\
\text { rating scale } \\
\text { of sore } \\
\text { throat }\end{array}$ & $24 \mathrm{~h}$ & 1 to 10 & $5(5,6)$ & $5(3,6)$ & $5(3,8)$ & $5(5,6)$ & 0.56 \\
\hline
\end{tabular}

\section{CONCLUSIONS}

ETT cuff pressure significantly increased after retractor replacement during ACDF. To adjust and maintain the ETT cuff pressure at $20 \mathrm{~mm} \mathrm{Hg}$, after cervical retractor was applied, reduced the severity of dysphagia and hoarseness. Increasing of cuff pressure was not caused by nitrous oxide so that it could be used. 